\title{
ESTUDO CINÉTICO DO PROCESSO SFS EM BIORREATOR PARA PRODUÇÃO DE ETANOL A PARTIR DE BAGAÇO DE CANA-DE-AÇÚCAR
}

\author{
P. V. F. DANTAS ${ }^{1}$,L. P. DE SOUZA ${ }^{1}$, A. DE A. GUILHERME ${ }^{1}$, E. S. DOS SANTOS ${ }^{1}$, F. A. \\ N. FERNANDES ${ }^{2}$, G. R. DE MACEDO ${ }^{1}$ \\ ${ }^{1}$ Universidade Federal do Rio Grande do Norte - Departamento de Engenharia Química \\ ${ }^{2}$ Universidade Federal do Ceará - Departamento de Engenharia Química \\ Contato: alexandredearaujoguilherme@gmail.com
}

\begin{abstract}
RESUMO:Devido a questões econômicas e ambientais, a substituição da matriz energética do petróleo pela biomassa vegetal torna-se importante no cenário mundial. Este trabalho teve como objetivo um estudo cinético do processo de sacarificação e fermentação simultânea(SFS) visando a otimização da produção de etanol em termos de produtividade e custo com enzimas. A levedura Saccharomycescerevisiae PE-2 foi utilizada como agente de fermentação nas quantidades de 0,5, 1,0 e 2,0 de inoculo, e a enzima usada foi a NS22086(Novozymes) com atividade inicial de 10, 15 e $20 \mathrm{FPU} / \mathrm{g}$ celulose e a quantidade de celulose foi de 2 a $6 \%$ contida no bagaço de cana. O bagaço foi pré-tratado com ácido sulfúrico em uma primeira etapa e hidróxido de sódio em uma segunda etapa. Os ensaios em biorreator foram em dorna de $2 \mathrm{~L}$ com $1 \mathrm{~L}$ de volume reacional, $\mathrm{pH}$ 5,0 \pm 0,2 e bagaço de cana como única fonte de carbono e energia. Desta forma, foi possível obter o processo otimizado partindo de 1,0 g/L de inoculo e $15 \mathrm{FPU} / \mathrm{g}$ celulose da enzima celulase e $6 \%$ de celulose inicial, obtendo uma produtividade de $1,75 \mathrm{~g} / \mathrm{Lh}$ em 18 horas de processo.
\end{abstract}

\section{INTRODUÇÃO}

A biomassa vegetal rica em material lignocelulósico está sendo estudada no mundo todo como uma fonte renovável de carbono e energia. Sem dúvida alguma, o etanol é o mais importante produto final obtido pela fermentação de hidrolisados lignocelulósicos, porém outros produtos também podem ser obtidos.

Para a produção de etanol de segunda geração (etanol celulósico) é preciso que se obtenham monômeros de carboidratos como a glicose proveniente da estrutura da parede celular vegetal, lignocelulose. Desta forma, diferentemente do processo convencional, para a produção de etanol de segunda geração é preciso que o material lignocelulósico seja submetido a um pré-tratamento químico, físico, biológico ou combinado e a uma hidrólise enzimática para que se tenha substrato disponível para a fermentação alcoólica.

Os pré-tratamentos são utilizados para se aumentar a conversão do material lignocelulósico em açúcares redutores como celobiose e glicose (Sun e Cheng, 2002) através 
do processo de hidrólise enzimática. Eles atuam desestruturando a matriz lignocelulósica, reduzindo as quantidades de lignina e hemicelulose e modificando a estrutura cristalina da celulose de forma a deixá-la mais susceptível ao ataque enzimático (Silversteinet al., 2007).

Pré-tratamentos ácidos envolvendo ácido sulfúrico, nítrico ou clorídrico podem solubilizar parte da hemicelulose expondo ainda mais a celulose ao ataque enzimático (Schellet al., 2003). Pré-tratamentos alcalinos se referem ao uso de soluções alcalinas como hidróxido de sódio, dentre outras, usadas basicamente para remover lignina e diminuir o grau de cristalinidade da celulose (Chang e Holtzapple, 2000).

$\mathrm{Na}$ hidrólise enzimática da celulose são necessário pelo menos três tipos de enzimas para se que se obtenha glicose. As endoglicanases (EG) (EC.3.2.1.4) hidrolisam as moléculas de celulose de forma randômica produzindo oligômeros. As celobiohidrolases ou exoglicanases $(\mathrm{CBH})$ (EC.3.2.1.91) convertem os oligômeros, formados pela ação das endoglicanases, em celobiose e celodextrinas solúveis. A $\beta$-glicosidase (BG), também chamada de celobiase, (EC.3.2.1.21) converte celobiose e celodextrinas em glicose (Zhouet al., 2009).

A celulose pode ser hidrolisada, por enzimas celulases, em glicose, e esta, pode ser fermentado para produzir etanol (Yang et al., 2011) em um processo com duas etapas. Entretanto, estes dois processos podem ser realizados em uma só etapa denominada de sacarificação e fermentação simultânea (SFS) (Vasquez et al., 2007) objetivando redução de tempo e energia diminuído custos de processo.

Este trabalho teve como objetivo um estudo cinético em biorreator do processo SFS variando as concentrações de inoculo, enzimas iniciais e celulose contida no bagaço de canade-açúcar visando a otimização da produção de etanol em termos de produtividade e custo com enzimas.

\section{MATERIAL E MÉTODOS}

\subsection{Enzimas, levedura e bagaço de cana-de-açúcar}

As enzimas utilizadas neste trabalho foram (NS22086) que corresponde às celulases cedidas pela Novozymes (Bagsvaerd, Dinamarca).O substrato utilizado foi o bagaço de canade-açúcar cedido gentilmente pela Usina Estivas (Arês - RN, Brasil) e a levedura utilizadafoi uma linhagem capaz de produzir etanol a partir de glicose, a Saccharomycescerevisiae PE- 2 (Basso et al., 2008).

\subsection{Estoque de leveduras, pré-inoculo e inoculo}

A levedura foi mantida, durante os ensaios, a $-20{ }^{\circ} \mathrm{C}$ em glicerol estéril sendo o estoque renovado a cada seis meses segundo Silva et al., (2008). Também foi mantida em meio YEPD inclinado a $4{ }^{\circ} \mathrm{C}$ sendo este estoque renovado a cada dois meses. 
Para a produção do pré-inoculo e inoculo foi utilizado o meio de cultura nas seguintes proporções; glicose $(30,0 \mathrm{~g} / \mathrm{L})$, extrato de levedura $(5,0 \mathrm{~g} / \mathrm{L}),\left(\mathrm{NH}_{4}\right)_{2} \mathrm{SO}_{4}(10,0 \mathrm{~g} / \mathrm{L}), \mathrm{KH}_{2} \mathrm{PO}_{4}$ (4,5 g/L), $\mathrm{MgSO}_{4} .7 \mathrm{H}_{2} \mathrm{O}(1,0 \mathrm{~g} / \mathrm{L})$ e $\mathrm{ZnSO}_{4}(0,65 \mathrm{~g} / \mathrm{L})$.

Para o preparo do pré-inoculo foi utilizado erlenmyers de $250 \mathrm{~mL}$ com $100 \mathrm{~mL}$ de meio de cultura, velocidade de agitação de $150 \mathrm{RPM}$, temperatura de $30{ }^{\circ} \mathrm{C}$. Uma colônia da levedura, crescida em placa de Petri, foi transferida para os frascos de erlenmyers onde foi incubada por 23 horas.

A partir do pré-inoculo, foi transferido $5 \mathrm{~mL}$ da suspensão celular para os meios de inoculo. As condições de processos foram iguais às do pré-inoculo com exceção da temperatura que foi de $35^{\circ} \mathrm{C}$ e o tempo de incubação que foi de 10 horas.

\subsection{Pré-tratamento no bagaço de cana}

O pré-tratamento ácido alcalino foi realizado utilizando bagaço na quantidade de sólidos de $20 \%(\mathrm{p} / \mathrm{v})$ imerso em uma solução de ácido sulfúrico $2 \%$ (v/v) submetido a uma temperatura de $121^{\circ} \mathrm{C}$ por 30 minutos, segundo Guoet al., (2009). Em seguida, a fração sólida foi lavada com água até o valor do $\mathrm{pH}$ ficar próximo ao $\mathrm{pH}$ da água de lavagem. Após o prétratamento ácido, o material foi submetido a um pré-tratamento alcalino para a deslignificação onde foi utilizada uma quantidade de $20 \%$ (p/v) do material sólido submerso em solução de hidróxido de sódio $4 \%(\mathrm{p} / \mathrm{v})$ submetido a uma temperatura de $121^{\circ} \mathrm{C}$ por 30 minutos, segundo Vasquez et al., (2007). Em seguida, o pH foi corrigido para 7,0 utilizando ácido clorídrico para posterior lavagem. O pré-tratamento visa à remoção de parte da hemicelulose (Schellet $a l .$, 2003), lignina e redução do grau de cristalinidade da celulose (Chang e Holtzapple, 2000).

\subsection{Cinéticaem biorreator}

O biorreator utilizado nesta etapa foi uma adaptação de uma dorna de 2 L (ApplikonDependableInstruments, Holanda) com controle de agitação (mod P100/ADI 1032, Applikon, Holanda) acoplados a uma torre de controle de $\mathrm{pH}$, nível e nutriente (mod TEC BIO, Tecnal, São Paulo/Brasil) usando um banho termostático para controle de temperatura (mod TE - 184, Tecnal, São Paulo/Brasil).

O estudo cinético foi realizado com $1 \mathrm{~L}$ de volume reacional.As quantidades foram de 0,5, 1,0 e 2,0 g/L de inoculo, a atividade enzimática inicial foi de 10, 15 e $20 \mathrm{FPU} / \mathrm{g}$ de celulose e a quantidade de celulose foi de 2 a $6 \%$ contida no bagaço de cana. $\mathrm{O}$ meio de cultura foi;extrato de levedura $(4,0 \mathrm{~g} / \mathrm{L}),\left(\mathrm{NH}_{4}\right)_{2} \mathrm{SO}_{4}(2,0 \mathrm{~g} / \mathrm{L}), \mathrm{KH}_{2} \mathrm{PO}_{4}(2,0 \mathrm{~g} / \mathrm{L})$, $\mathrm{MgSO}_{4} \cdot 7 \mathrm{H}_{2} \mathrm{O}(0,75 \mathrm{~g} / \mathrm{L})$ e a celulose contida no bagaço de cana como fonte de carbono e energia. Em ensaios anteriores verificou-se que as enzimas celulases utilizadas aqui não necessitam de uma suplementação com enzimas do tipo $\beta$-glicosidase. 
Amostras foram coletadas em intervalos de tempo determinados por 24 horas para análises de açúcares celobiose, glicose, xilose e celulose, bem como, a produção de etanol e glicerol e se fazer o monitoramento do crescimento microbiano.

\subsection{Ensaios analíticos}

As quantificações dos açúcares, etanol, glicerolforam feitas por CLAE usando a coluna Shim-pack SCR 101-H (SHIMADZU, Kyoto/Japão) a $65^{\circ} \mathrm{C}$ e ácido sulfúrico $5 \mathrm{mM}$ em água MiliQ (D7031, BarnsteadEasyPure RF System - Iowa/EUA) como fase móvel, uma vazão de 0,6 mL/min e o índice de refração (RID-10A, SHIMADZU, Japão) como detector.

A determinação de celulose consumida foi de acordo com a NREL (Dowe e McMillan, 2001).

A determinação da biomassa celular para padronizar o inoculo foi realizada fazendo-se uma correlação entre massa seca em g/L e ABS em espectrofotômetro. Para o estudo do processo SFS, foi feita uma correlação entre a massa seca em $\mathrm{g} / \mathrm{L}$ e contagem de células viáveis em placa UFC/mL, uma vez que, a bagaço de cana é um interferente na leitura de ABS.

\section{RESULTADOS E DISCUSSÃO}

Em um primeiro ensaio com 0,5 g/L de inoculo, $15 \mathrm{FPU} / \mathrm{g}$ de celulose das enzimas celulases e variando a celulose contida no bagaço de cana de 2 a $6 \%$ foi observado que o maior crescimento celular foi no ensaio partindo de $4 \%$ de celuloseobtendo-se 3,95 g/L de biomassa em 12 horas, entretanto, a maior produção de etanol foi no ensaio partindo de $6 \%$ de celulose, onde o crescimento celular foi menor, 3,78 após 18 horas, mas o etanol produzido foi de 29,45 g/L. A partir destes resultados, novos ensaios foram realizados variando-se a quantidade de inoculo e atividade enzimática inicial mantendo-se a quantidade de celulose em 6\%. Nas Figuras 1 e 2 estão apresentados os resultados de crescimento microbiano e produção de etanol para os ensaios realizados variando inoculo e atividade enzimática inicial.

Pode-se observar que os ensaios com 10 e 15 FPU/g celulose de atividade enzimática inicial e $0,5 \mathrm{~g} / \mathrm{L}$ de inoculo ficaram com resultado próximos em biomassa mas não para a produção de etanol que foi maior no ensaio partindo de 15 FPU. Já o ensaio partindo de 20 FPU e 0,5 g/L de inoculo produziu mais biomassa que os anteriores, mas, a mesma quantidade de etanol que o processo partindo com 15 FPU em 24 horas, entretanto, as produtividades foram maiores entre 8 e 18 horas de processo. Nos ensaios partido de 15 FPU e 1,0 e 2,0 g/L de inoculo, não se observa uma maior produção de biomassa nem tão pouco de etanol quando comparado aos ensaios anteriores. Pode-se observar que se reduzindo a atividade enzimática de $20 \mathrm{FPU}$ para $15 \mathrm{FPU} / \mathrm{g}$ celulose e aumentando o inoculo de 0,5 para 
1,0, se obtêm a mesma produtividade em etanol. Já, se aumentar o inoculo de 1,0 para 2,0 g/L não se observa diferença de produtividade devido à limitações na produção de glicose pelo processo de hidrólise enzimática.

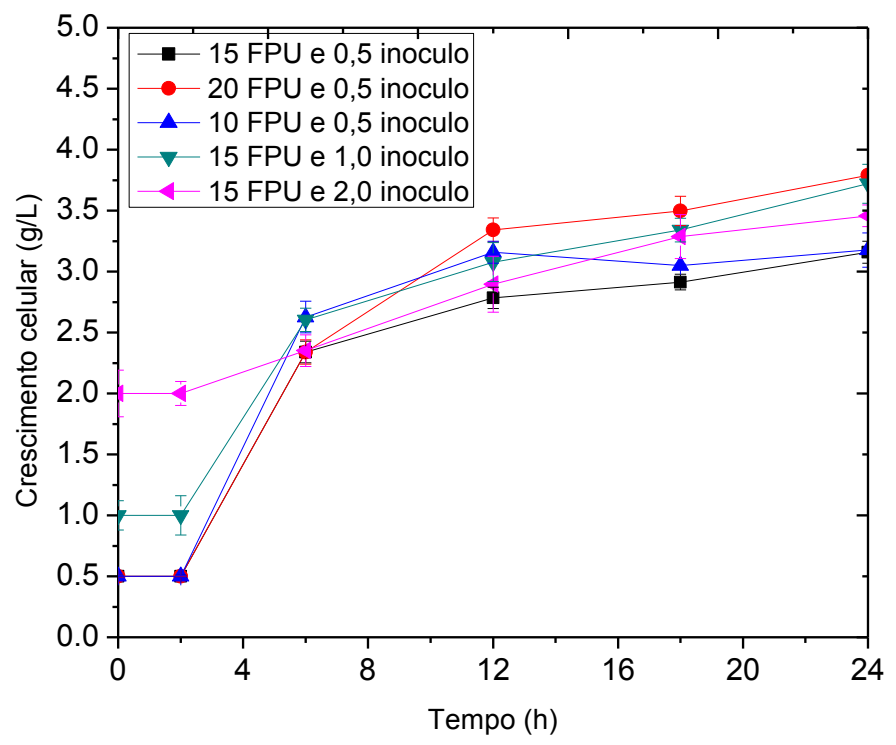

Figura 1 - Crescimento celular.

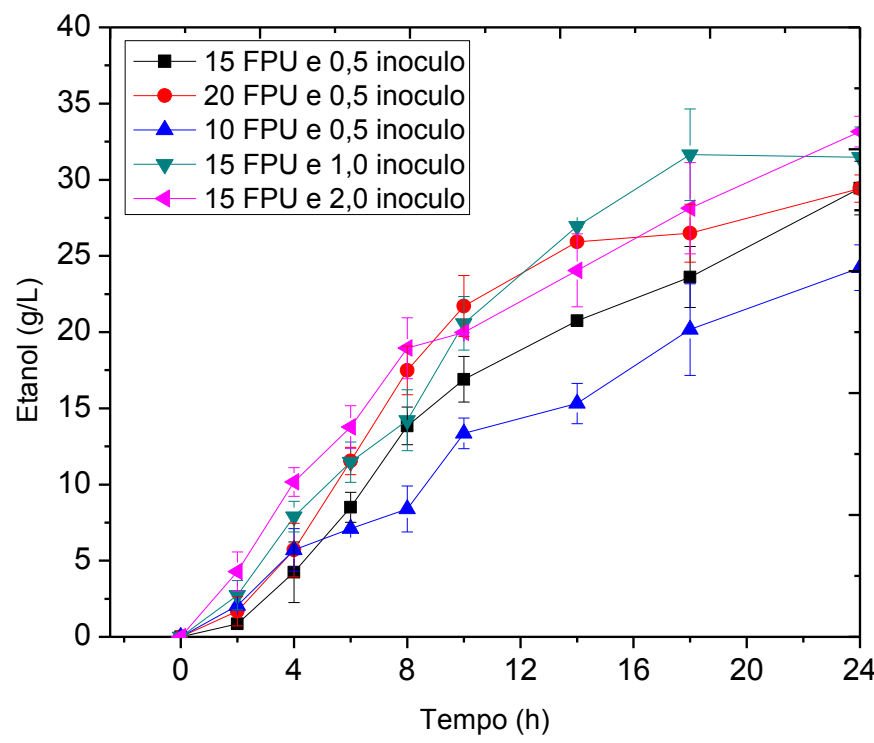

Figura 2 - Produção de etanol. 
$\mathrm{Na}$ Tabela 1 estão apresentados os resultados de produção de etanol e glicerol, a celulose residual do processo, o rendimento de etanol por grama de bagaço utilizado e o rendimento teórico do processo.

Tabela 1 - Produção de etanol e glicerol, celulose residual, rendimento de etanol por grama de bagaço utilizado e rendimento teórico do processo.

\begin{tabular}{cccccc}
\hline $\begin{array}{c}\text { Condição } \\
\text { inicial }\end{array}$ & $\begin{array}{c}\text { Glicerol } \\
(\mathrm{g} / \mathrm{L})\end{array}$ & Etanol $(\mathrm{g} / \mathrm{L})$ & $\begin{array}{c}\text { Etanol } \\
(\mathrm{g} / \mathrm{g} \\
\text { bagaço })\end{array}$ & $\begin{array}{c}\text { Celulose } \\
\text { residual } \\
(\mathrm{g} / \mathrm{L})\end{array}$ & $\begin{array}{c}\text { Rendimento } \\
\text { teórico }(\%)\end{array}$ \\
\hline $\mathrm{a}$ & $0,95 \pm 1,54$ & $29,45 \pm 1,56$ & $0,31 \pm 0,01$ & $8,01 \pm 0,54$ & 86,63 \\
$\mathrm{~b}$ & $1,24 \pm 0,08$ & $29,42 \pm 2,30$ & $0,31 \pm 0,00$ & $8,07 \pm 0,06$ & 86,54 \\
$\mathrm{c}$ & $0,52 \pm 0,04$ & $24,22 \pm 1,56$ & $0,26 \pm 0,01$ & $17,24 \pm 1,09$ & 71,25 \\
$\mathrm{~d}$ & $1,21 \pm 0,1$ & $31,47 \pm 2,09$ & $0,34 \pm 0,03$ & $4,45 \pm 0,93$ & 92,58 \\
$\mathrm{e}$ & $1,37 \pm 0,01$ & $33,15 \pm 1,95$ & $0,35 \pm 0,00$ & $1,47 \pm 0,25$ & 97,53 \\
\hline
\end{tabular}

$\mathrm{a}-15 \mathrm{FPU} / \mathrm{g}$ celulose e $0,5 \mathrm{~g} / \mathrm{L})$ de inoculo, b - 20 FPU/g celulose e 0,5 (g/L) de inoculo, c - 10 FPU/g celulose e $0,5(\mathrm{~g} / \mathrm{L})$ de inoculo, $\mathrm{d}-15 \mathrm{FPU} / \mathrm{g}$ celulose e $1,0(\mathrm{~g} / \mathrm{L})$ de inoculo, e - 15 FPU/ g celulose e 2,0 (g/L) de inoculo.

Apesar de o rendimento teórico no processo partindo de 2,0 g/L de inoculo ser um pouco maior que o processo partindo de $1,0 \mathrm{~g} / \mathrm{L}$, a produtividade do segundo foi maior em 18 horas de processo que foi de 1,75 g/L.h comparado com 1,56 g/L.h no processo partindo de 2,0 g/L de inoculo. Na Figura 3 estão apresentados os resultados cinéticos para os açúcares, produção de etanol e biomassa celular durante o processo SFS partindo com 1,0 g/L de inoculo, $15 \mathrm{FPU} / \mathrm{g}$ celulose e $6 \%$ de celulose contida no bagaço de cana.

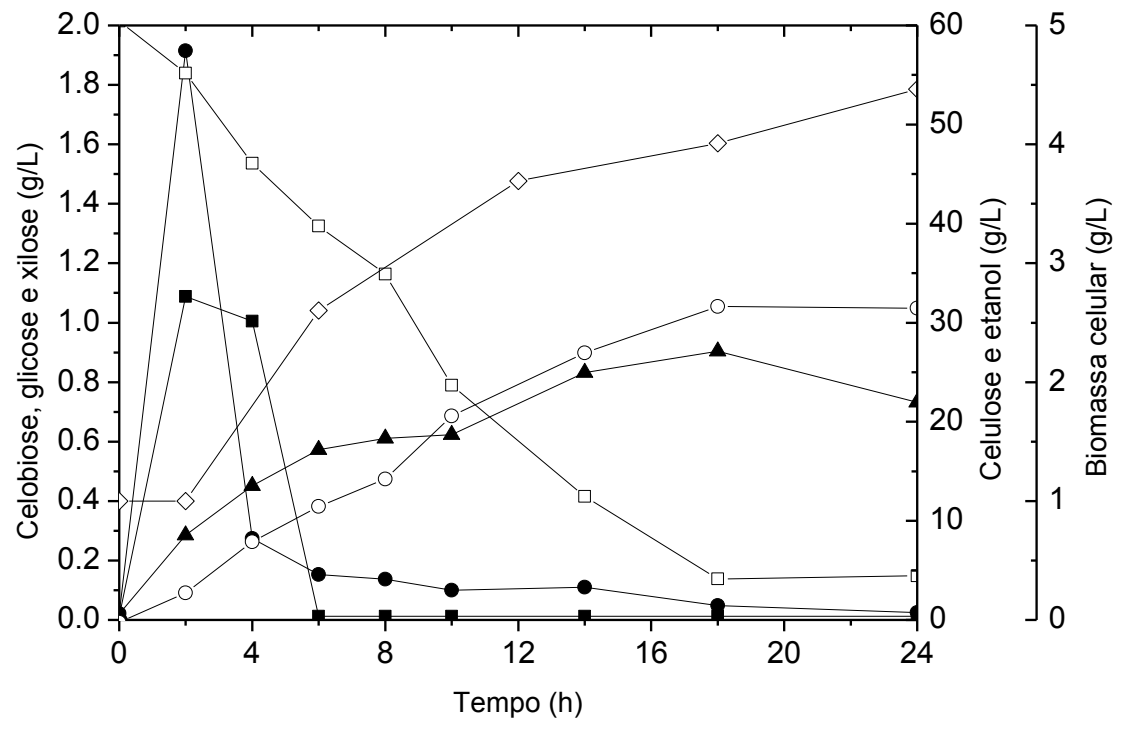

-celobiose, •-glicose, $\boldsymbol{\Delta}$-xilose, $\square$-celulose, o-etanol e $\diamond$-biomassa celular.

Figura 3 - Perfil cinético do processo SFS otimizado. 
Pode-se observar que a celobiose é produzida em maior quantidade no início do processo, bem como, a glicose, porém com o início da fase Log da levedura, estes açúcares são consumidos rapidamente e ficando constantes próximos de zero no decorrer do processo. A xilose é liberada ao meio e ao que se parece não é consumida pela levedura. A celulose é consumida em $92,58 \%$ e a biomassa celular atinge $4,5 \mathrm{~g} / \mathrm{L}$ ao final de 24 horas de processo.Ohgrenet al (2007) produziu 21,4 g/L de etanol, 0,75 g etanol/grama de palha de milho e um rendimento de $81,5 \%$ em um reator de $15 \mathrm{~L}$ com $5 \mathrm{~kg}$ de peso de biomassa. Desta forma, os resultados deste trabalho estão em concordância com a literatura.

\section{CONCLUSÃO}

De acordo com os resultado apresentados neste trabalho, é possível concluir que o processo de sacarificação e fermentação simultânea aqui realizado em biorreator com $1 \mathrm{~L}$ de volume reacional, foi otimizado em relação à atividade enzimática inicial (15 FPU/g celulose), inoculo $(1,0 \mathrm{~g} / \mathrm{L})$ e celulose contido no bagaço de cana $(6 \%)$. Desta forma, foi possível reduzir custo com enzimas fazendo-se uso de inoculos maiores para aumentar a produtividade do processo obtendo-se excelentes rendimentos teóricos em etanol.

Um estudo com diferentes tipos de impelidores e frequência de agitação faz-se necessário quando se pensa em uma ampliação de escala deste processo. Desta forma, os resultados aqui apresentados são um ponto de partida para novos estudos do processo aqui apresentado.

Um estudo cinético em batelada alimentada deve ser feito com as condições aqui estudadas e otimizadas visando uma melhoria na agitação do sistema, bem como, uma redução ainda maior das enzimas utilizadas.

\section{REFERÊNCIAS}

BASSO, L. C.; AMORIM, H. V.; OLIVEIRA,A. J.; LOPES,M. L. Yeast selectionfor fuel ethanol production in Brazil, FEMS Yeast Res, v. 8, p. 1155-1163, 2008.

CHANG, V.; HOLTZAPPLE, M. Fundamental factors affecting biomass enzymatic reactivity.ApplBiochemBiotechnol, v. 84-86, p. 5-37, 2000.

DOWE, N.; McMILLAN, J. SSF Experimental Protocol -Lignocellulosic Biomass Hydrolysis and Fermentatio.Laboratory Analytical Procedure (LAP), Technical Report, NREL, 2001.p. 19. 
GUO, G. L.; HSU, D. C.; CHEN, W. H.; CHEN, W.H.; HWANG, W. S. Characterization of enzymatic saccharification for acid-pretreated lignocellulosic materials with different lignin composition. Enzyme MicrobTechnol, v. 45, p. 80-87, 2009.

OHGREN, K.; BURA, R.; LESNICKI, G.; SADDLER, J.; ZACCHI, G.A comparison between simultaneous saccharification and fermentation and separate hydrolysis and fermentation using steam-pretreated corn stover.Process Biochemistry,v. 42, p. 834-839, 2007.

SCHELL, D. J.; FARMER, J.; NEWMAN, M.; MCMILLAN, J. D. Dilutesulfuric acid pretreatment of corn stover in pilot-scale reactor - investigation of yields, kinetics, and enzymatic digestibilities of solids.Appl Biochem Biotechnol, v. 105, p. 69-85, 2003.

SILVA,J. O.; COSTA,P. P.; RECHE,S. H. C.Manutenção de leveduras por congelamento a $20^{\circ}$ C.RBAC, v. 40, n. 1, p. 73-74, 2008.

SILVERSTEIN, R. A.; CHEN, Y.; SHARMA-SHIVAPPA, R. R.; BOYETTE, M. D. J. O. A comparison of chemical pretreatment methods for improving saccharification of cotton stalks. BioresourTechnol, v. 98, p. 3000-3011, 2007.

SUN, Y.; CHENG, J. Hydrolysis of lignocellulosic materials for ethanol production: a review. Bioresour Technol, v. 83, p. 1-11, 2002.

VASQUEZ, M. P.; SILVA, J. N. C.; SOUZA, M. B. Jr.; PEREIRA, N. Jr. Enzymatic hydrolysis optimization to ethanol production by simultaneous saccharification and fermentation. ApplBiochemBiotechnol, v. 136-140, p. 141-154, 2007.

YANG,P.; GUO,L.; CHENG, S.; LOU, N.; LIN, J. Recombinant multi-functional cellulase activity in submerged fermentation of lignocellulosic wastes, Renewable Energy. v. 36, p. 3268-3272, 2011.

ZHOU, J.; WANG, Y. H.; CHU, J.; LUO, L. Z.; ZHUANG, Y. P.; ZHANG, S. L. Optimization of cellulase mixture for efficient hydrolysis of steam-exploded corn stover by statistically designed experiments. BioresourTechnol, v. 100, p. 819-825, 2009. 